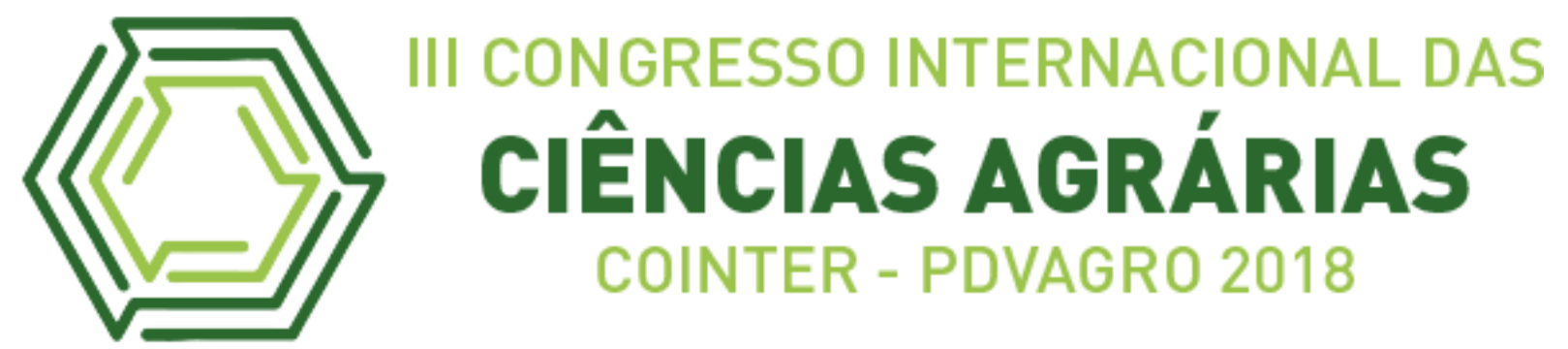

\title{
DESENVOLVIMENTO VEGETATIVO DA VINAGREIRA (Hibiscus Sabdariffa L.) EM FUNÇÃO DE DIFERENTES NÍVEIS DE pH
}

\section{VEGETABLE DEVELOPMENT OF VINAGREIRA (Hibiscus Sabdariffa L.) IN THE FUNCTION OF DIFFERENT pH LEVELS \\ Apresentação: Pôster}

\author{
Davi Belchior Chaves ${ }^{1}$; Ayrna Katrinne Silva do Nascimento ${ }^{2}$; Francisco Mateus \\ Medina Gomes ${ }^{3}$; Álvaro Itaúna Schalcher Pereira ${ }^{4}$
}

\section{DOI: https://doi.org/10.31692/2526-7701.IIICOINTERPDVAGRO.2018.00423}

\section{Introdução}

A vinagreira é uma planta pertencente à família das Malváceas, do gênero Hibisco, sendo conhecida popularmente como hibisco, hibiscus, rosela, groselha, azedinha, quiabo azedo, caruru-azedo, caruru-da-guiné e quiabo-de-angola (CARDOSO, 1997), essa cultura está presente no mundo todo, mas tem grande cultivo no nordeste Brasileiro, especificamente no maranhão, onde há o maior cultivo dessa cultura que assume grande importância no cenário alimentício como hortaliça folhosa (MINISTÉRIO DA SAÚDE, 2015).

Rico em vitaminas sendo também uma alternativa de alimentação saudável, adapta-se bem a regiões quentes e tropicais, tais características chamaram atenção da agricultura familiar por acentuar- se tão bem em uma região com solos secos e com níveis de pH tão distintos.

Segundo a EMBRAPA (2014), o nordeste destaca- se por ser o maior produtor de vinagreira, mas sofre com baixa produtividade devido à baixa fertilidade somada com solos ácidos e alcalinos. Diante do exposto e na escassez de estudos relacionados ao comportamento vegetativo dessa cultura em solos da região nordestina, objetivou- se avaliar o desenvolvimento vegetativo da vinagreira em função de diferentes níveis de pH que compões o solo dessa região.

\section{Fundamentação Teórica}

\footnotetext{
${ }^{1}$ Licenciatura em Ciências Agrarias, IFMA- Campus Codó, davidesigne123@gmail.com

${ }^{2}$ Licenciatura em Ciências Agrarias, IFMA-Campus Codó, ayrna15@hotmail.com

3 Bacharelado em Agronomia, IFMA-Campus Codó, fcomateusmedinagomes@gmail.com

${ }^{4}$ Doutor. Professor do IFMA- Campus Codó,
} 
Na visão de Cardoso (1997), a vinagreira é uma planta da família das Malváceas, do gênero Hibisco, que existem mais de 190 espécies de plantas com vários nomes populares sendo eles hibisco, hibiscus, rosela, groselha, azedinha, quiabo azedo, caruru-azedo, caruruda-guiné e quiabo-de-angola. Existem muitas controvérsias sobre a origem dessa espécie, mas conforme Panizza (1997), a cultura veio da África e Ásia pelo tráfico negreiro, desembarcando no nordeste brasileiro, que tornou- se a maior produtora de vinagreira do Brasil. Por vir de regiões com ambiente parecidos com o do nordeste, para Rodrigues (2008), essa característica faz com a vinagreira se encaixe bem no perfil climático do nordeste brasileiro, por esse motivo vem sendo muito cultivada. Um solo com nutrientes e $\mathrm{pH}$ adequado é essencial para uma boa produtividade e na visão de Mcclintock (2004), a vinagreira consegue adaptar- se a solos com pH que varia de 4,5 a 8. Esses níveis são propícios à perca de nutrientes por lixiviação, apesar de que a vinagreira absorve relativamente pequenas quantidades de nutrientes, quando comparadas com outras culturas, mas por ser uma hortaliça folhosa, é considerada exigente em nutrientes, em resposta de seu ciclo relativamente curto (NOVAIS, 1999). Essa exigência de muito nutriente pode ser um problema para a cultura obter uma ótima produção, pois o nordeste destaca- se por ser o maior produtor de vinagreira, mas apresenta baixa produtividade devido baixa fertilidade somada com elevada capacidade de retenção de fósforo e solos muito alcalino e ácido (EMBRAPA, 2014).

\section{Metodologia}

O experimento foi conduzido no Instituto Federal de Ciências e Tecnologia do Maranhão campus Codó, durante 135 dias. Baseando-se na metodologia de (RUARO et.al 2010), os níveis de pH utilizados foram: T1- 3,3; T2- 4,7; T3- 5,6; T4- 6,3 e T5- 7,8. Para a obtenção das quantidades dos diferentes níveis de $\mathrm{pH}$, utilizou- se crescentes doses de calcário misturadas a determinadas quantidades de solo incubado. As doses de calcário utilizadas na incubação seguiram uma ordem crescente correspondente a $0 ; 25 ; 50 ; 100 ; 150 ; 20 ; \mathbf{e} 350$ Kg $\mathbf{m}^{2}$. A unidade experimental era constituída de um vaso de plástico com capacidade para 30L contendo $30 \mathrm{~kg}$ de solo, utilizando delineamento em blocos casualizados (DBC) com cinco tratamentos e cinco repetições.

O calcário utilizado foi calcítico, com Poder Relativo de Neutralização de 104,5\%. Após a aplicação do calcário, o pH do solo foi monitorado a cada 2 dias, até a estabilização 
dos valores, que ocorreu aos 60 dias. Durante esse período, a umidade do solo foi mantida na capacidade de campo, pela adição de água destilada a cada 48 hora. Após o período de incubação, amostras de $20 \mathrm{~g}$ de solo foram retiradas para compor uma amostra composta por tratamento. Tais amostras foram submetidas à análise para determinação dos valores de $\mathrm{pH}$ e para a elaboração da curva de calibração. A partir dos valores de $\mathrm{pH}$ obtidos, indicou a necessidade de calagem de $0 ; 6,0 ; 12,0$ e 21,0 g de calcário. 2,0 kg $\mathrm{kg}^{-1}$ de solo, o necessário para se atingir o $\mathrm{pH}$ de 3,3;4,7;5,6; 6,3 e 7,8 (Tabela1). As mudas de vinagreira foram transplantas 60 dias após aplicação das doses de calcário.

A adubação de $\mathbf{N}$ e $\mathbf{K}_{\mathbf{2}} \mathbf{O}$ foram feitas com base na metodologia de Batista Neto (2017), onde aplicou- se de forma parcelada $\mathbf{8 0 K g}$ há $^{-1}$ de $\mathrm{K}_{2} 0$ e $\mathbf{1 8 0 K g}$ há $^{-1}$ de $\mathrm{N}$, sendo 1/3 aplicado no transplantio e o restante aplicado 50 dias após o transplantio. A adubação de $\mathbf{P}_{\mathbf{2}} \mathbf{O}_{\mathbf{5}}$ foi feita conforme a metodologia de Nascimento (2018), houve a aplicação de $\mathbf{5 0 K g}$ há ${ }^{-1}$ de $\mathrm{P}_{2} \mathrm{O}_{5}$ em dose única na hora do transplantio. As fontes utilizadas para obtenção desses nutrientes foram Cloreto de Potássio, Super Fosfato Triplo e Ureia.

(Tabela1): Características do solo utilizado na avaliação da vinagreira em diferentes níveis de pH após ser incubado com calcário. Fonte: própria

\begin{tabular}{|c|c|c|c|c|c|c|c|c|c|c|c|c|}
\hline pH & $\mathbf{A l}^{3}$ & $\mathbf{H}+\mathbf{A l}$ & $\mathrm{Ca}^{2}+\mathrm{Mg}^{2}$ & $\mathrm{Ca}^{2}$ & $\mathbf{K}^{1}$ & $\mathbf{T}$ & $\mathbf{P}$ & M.O & $\mathbf{V}$ & $\begin{array}{c}\text { Areia } \\
\text { total }\end{array}$ & Silte & Argila \\
\hline $\mathrm{CaCl}^{3}$ & & & Cmol dm & & & & $\begin{array}{l}\text { Mg. } \\
\text { dm }^{3}\end{array}$ & $\begin{array}{c}\text { g.dm } \\
3\end{array}$ & $\%$ & $\%$ & $\%$ & $\%$ \\
\hline 3,3 & 3,5 & 12,3 & 0,87 & 0,2 & 0,18 & 9,3 & 0,6 & 27,3 & 0,5 & 28 & 23 & 50 \\
\hline 4,7 & 1,98 & 10,1 & 1,6 & 0,8 & 0,20 & 11,7 & 0,6 & 31,4 & 11 & 28 & 23 & 50 \\
\hline 5,6 & 0,0 & 4,3 & 9,1 & 5,3 & 0,21 & 15,3 & 0,6 & 30,4 & 69 & 28 & 23 & 50 \\
\hline 6,3 & 0,0 & 3,2 & 11,4 & 6,4 & 0,20 & 15,6 & 0,6 & 33,5 & 79 & 28 & 23 & 50 \\
\hline 7,8 & 0,0 & 1,87 & 15,6 & 9,0 & 0,16 & 18,2 & 0,3 & 31,3 & 89 & 28 & 23 & 50 \\
\hline
\end{tabular}

As mudas de vinagreira foram produzidas na casa de vegetação do IFMA, utilizando esterco bovino e terra preta na proporção 2:1, e colocados em copos de poliestireno de $350 \mathrm{ml}$. Utilizou-se semente de vinagreira comum, comprada com os produtores da região, 30 dias depois do plantio das sementes, as mudas estavam com $15 \mathrm{~cm}$ de tamanho e foram transplantas para os vasos experimentais. Após 100 dias as plantas foram retiradas para obtenção das diferentes variáveis. O material coletado foi conduzido ao Laboratório de Solos presente no IFMA, para que as seguintes características fossem analisadas: diâmetro do caule (DC); 
tamanho da raiz (TR); matéria fresca da parte aérea (MFPA); matéria seca da parte aérea (MSPA); altura da planta (AP); quantidade de folhas (QF) e peso total da planta (PTP).

\section{Resultados e Discussões}

Conforme a análise de variância (Tabela 2), não houve nenhuma significância entre as variáveis analisadas MF, MS, QF, DC, PTP relacionado aos blocos, apenas a variável AP teve significância em função dos diferentes níveis de pH. A variância em relação aos tratamentos, apenas na variável DC não apresentou significância dentre as demais avaliadas

Tabela 2. Resumo da análise de variância para as variáveis, matéria fresca (MF) e seca (MS), quantidade de folhas (QF), diâmetro do caule (DC), peso total da planta (PTP), tamanho da planta (TP) e o coeficiente de variação $(\mathrm{CV})$ em função de diferentes níveis de $\mathrm{pH}$. Fonte: própria

\begin{tabular}{cccccccc}
\hline & GL & MF & MS & QF & DC & PTP & AP \\
\hline & & & & QUADRADO MÉDIO & & \\
BLOCO & 4 & $1542.860^{\mathrm{ns}}$ & $130.141^{\mathrm{ns}}$ & $500.100^{\mathrm{ns}}$ & $2.990^{\mathrm{ns}}$ & $5604.670^{\mathrm{ns}}$ & $0.01743^{*}$ \\
TRATAMENTO & 4 & $6684.660^{*}$ & $189.140^{*}$ & $1911.360^{*}$ & $13.740^{\mathrm{ns}}$ & $6035.060^{*}$ & $0.01140^{*}$ \\
RESÍDUO & 16 & 1761.310 & 39.990 & 244.010 & 1.680 & 17534.985 & 0.00632 \\
TOTAL & 24 & - & - & - & - & - & - \\
CV\% & - & $\mathbf{1 5}$ & $\mathbf{1 1 , 9 2}$ & $\mathbf{9 , 6 5}$ & $\mathbf{8 , 7 4}$ & $\mathbf{1 2 , 4 8}$ & $\mathbf{5 , 8 7}$ \\
\hline
\end{tabular}

** significativo ao nível de $1 \%$ de probabilidade $(\mathrm{p}<.01)$, * significativo ao nível de $5 \%$ de probabilidade $(.01$ $=<\mathrm{p}<.05),{ }^{\mathrm{ns}}$ não significativo $(\mathrm{p}>=.05)$.

Na variável altura da planta (AP) (Tabela 3), a cultura não obteve boa resposta segundo o teste $\mathrm{T}$ quando submetidos ao $\mathrm{pH}$ de 3,3 e 7,8 com médias de 1,60 e 164 metros. Enquanto que nos tratamentos $\mathrm{T} 3=5,6$ e T4=6,3 obteve- se as melhores médias com um ganho de altura de 6,8\% em comparação ao T1, demostrando uma possível sensibilidade da cultura da vinagreira a solos muito ácidos e alcalinos. Ariati (2015) observou no seu experimento de alface submetidas a pH que quanto mais o meio era ácido ou alcalino a cultura tinha uma queda em relação ao crescimento, para Malavolta (1980) nutrientes de suma importância para o desenvolvimento da planta só estão em ótima disponibilidade com $\mathrm{pH}>5$ e $<7,3$.

Na variável matéria fresca (MF) (Tabela 3), o T3=5,6 e T4=6,3 obtiveram as melhores médias segundo teste T, com percentual de 37,2\% de MF a mais que o T1, enquanto os T1 $=3,3$ e T5 $=7,8$ obtiveram as piores médias: 153,8 e 162,8 g. Muitos desses resultados tem ligação direta com as médias da variável quantidade de folha $(\mathrm{QF})$, onde nos tratamentos T3 e T4 obteve- se as maiores quantidades de folhas por planta 38,5\% a mais de folhas em comparação ao T1 que obteve a pior quantidade 153 folhas/p, tendo influência direta nos resultados da variável MF. Esse efeito na quantidade de folhas foi observado por Ariati (2015), conforme o meio estava ácido o número de folhas por plantas diminuía. Malavolta (1980) 
explica que culturas em meios ácidos $\mathrm{pH}<5$ tende a ter poucas folhas, é uma tática da planta já que nesse nível de $\mathrm{pH}$ não a disponibilidade de nutrientes suficiente para um ápice vegetativo.

Tabela 3: Comparação entre os testes de médias do tamanho da planta (TP), matéria fresca (MF), matéria seca (MS), diâmetro do caule (DC), peso total da planta (PTP) e quantidade de folhas (QF). Fonte: própria

\begin{tabular}{|c|cccccc|}
$\begin{array}{c}\text { TRATAMENTOS } \\
(\text { KG HA-1 })\end{array}$ & $\begin{array}{c}\text { AP } \\
(\mathbf{M})\end{array}$ & $\begin{array}{c}\text { MF } \\
(\mathbf{G})\end{array}$ & $\begin{array}{c}\text { MS } \\
(\mathbf{G})\end{array}$ & $\begin{array}{c}\text { DC } \\
(\mathbf{M M})\end{array}$ & $\begin{array}{c}\text { PTP } \\
(\mathbf{G})\end{array}$ & QF \\
\hline T1- 3,3 & $1,60^{\mathrm{c}}$ & $153,8^{\mathrm{c}}$ & $29,2^{\mathrm{c}}$ & $17,6^{\mathrm{a}}$ & $614,6^{\mathrm{c}}$ & $153,6^{\mathrm{c}}$ \\
\hline T2- 4,7 & $1,68^{\mathrm{b}}$ & $170,8^{\mathrm{b}}$ & $35,0^{\mathrm{b}}$ & $17,7^{\mathrm{a}}$ & $782,0^{\mathrm{b}}$ & $187,0^{\mathrm{b}}$ \\
\hline T3- 5,6 & $1,71^{\mathrm{a}}$ & $210,6^{\mathrm{a}}$ & $48,0^{\mathrm{a}}$ & $17,9^{\mathrm{a}}$ & $938,4^{\mathrm{a}}$ & $212,4^{\mathrm{a}}$ \\
T4- 6,3 & $1,70^{\mathrm{a}}$ & $200,4^{\mathrm{a}}$ & $45,8^{\mathrm{ab}}$ & $17,9^{\mathrm{a}}$ & $897,4^{\mathrm{ab}}$ & $209,4^{\mathrm{a}}$ \\
T5- 7,8 & $1,64^{\mathrm{bc}}$ & $162,8^{\mathrm{bc}}$ & $33,8^{\mathrm{b}}$ & $17,9^{\mathrm{a}}$ & $688,2^{\mathrm{bc}}$ & $185,0^{\mathrm{b}}$
\end{tabular}

As médias seguidas pela mesma letra não diferem estatisticamente entre si. Foi aplicado o Teste de Tukey ao nível de $5 \%$ de probabilidade.

$\mathrm{Na}$ variável matéria seca (MS), o tratamento T3 se sobressaiu dentre os demais tratamentos obtendo um acréscimo de $65 \%$ de matéria seca em relação a pior média, que foi o tratamento $\mathrm{T} 1$ obtendo apenas $29 \mathrm{~g}$ de $\mathrm{MS}$, enquanto os T2, T4 e T5 obtiveram resultados medianos e um acréscimo de 30\% de MS em relação ao T1. Essas médias obtidas tem ligação direta com as médias da variável MF.

No variável diâmetro do caule (DC), os tratamentos não diferenciaram estatisticamente, no experimento de Nascimento (2018), foi observado que a cultura da vinagreira obteve uma média de $17 \mathrm{~cm}$ de $\mathrm{DC}$ em um $\mathrm{pH}=5,4$, enquanto Batista Neto (2017) observou uma média de $18 \mathrm{~cm}$ em $\mathrm{pH}=7,3$. Esses valores demostram que o nível de $\mathrm{pH}$ não afeta o desenvolvimento do caule da vinagreira nas condições do experimento.

No variável peso total da planta PTP, o tratamento T3 obteve a melhor média dentre os demais tratamentos, um acréscimo de 52,6\% de PTP em comparação ao pior tratamento T1 que atingiu uma média de 614,6 g de PTP, os tratamentos T2 e T4 obtiveram resultados medianos. As variáveis DC, MF e QF influenciam diretamente nos resultados da variável PTP.

\section{Conclusões}

Nas condições em que foram conduzidas o experimento, concluiu-se que: os níveis de $\mathrm{pH}=5,6$ favoreceram no desenvolvimento da vinagreira e a cultura respondeu positivamente. 
Os níveis de $\mathrm{pH}=4,7$ e $\mathrm{pH}=6,3$ mostraram- se adequados para o cultivo da vinagreira, respondendo positivamente em todas variáveis estudadas.

Os níveis $\mathrm{pH}=3,3$ e $\mathrm{pH}=7,8$ foram os que demonstraram as menores médias, indicando uma possível sensibilidade da cultura a solos muito ácidos e alcalinos

\section{Referências}

ARIATI, Wagner Luiz; INTERAÇÃO ENTRE pH NA GERMINAÇÃO DE SEMENTE DE ALFACE (LACTUCA SATIVA). UNIVERSIDADE DO EXTREMO SUL CATARINENSE - UNESC CURSO DE CIÊNCIAS BIOLÓGICAS BACHARELADO, Criciúma,10 de JUNHO de 2015

BATISTA NETO, J. V. Avaliação das características vegetativas da Hibiscus sabdarifa L. submetida a diferentes doses de adubação nitrogenada de cobertura - COINTER 2017.

CARDOSO, M. O. (Coord.) (1997). Hortaliças não-convencionais da Amazônia. Brasília: Embrapa.

EMPRESA BRASILEIRA DE PESQUISA AGROPECUARIA. Manual de análise química de solo, planta e fertilizante. 2. Ed. Brasília: EMBRAPA informações tecnológicas, 2014.

MALAVOLTA, E. Elementos de nutrição mineral de plantas. São Paulo, Ceres, 1980. 251p.

MCCLINTOCK, N. C.; EL TAHIR, I. M. Hibiscus sabdariffa L. In: GRUBBEN, G. J. H.; DENTON, O. A. Ed. PROTA 2: Vegetables/Legumes. Wageningen, Netherlands: PROTA; 2004. [CD-Rom]

MINISTÉRIO DA SAÚDE. Alimentos regionais brasileiros. $2^{\mathrm{a}}$ ed. Brasília: MS- OS, 2015. $168 \mathrm{p}$.

NASCIMENTO, Ayrna Katrinne S.; Análise do desenvolvimento da vinagreira submetidas a diferentes doses de adubação fosfatada (pag. 155). Livro de Resumos expandidos aceitos no IV EMCA - Encontro Maranhense de Ciências Agrárias: Tecnologias sustentáveis e agroecológicas na produção florestal e agropecuária. De 25 a 29 Agosto de 2018 realizado por discentes da Universidade Estadual da Região Tocantina do Maranhão UEMASUL, Imperatriz - MA. ISSN: 2447-1429.

NOVAIS, R.F.; SMYTH, T.J. Fósforo em solo e planta em condições tropicais. ViçosaMG, 1999, 399 p.

PANIZZA, S. Plantas que curam: cheiro de mato. 18. Ed. São Paulo: IBRASA, 1997. 
RODRIGUES, S. DA G. G. A contemporaneidade da gastronomia Ludovicense: (Cuxá) X Big Mac/Mac Donald na cultura, identidade e tradição. Revista Cambiassu, v. 18, n. 4, p. $311-325,2008$.

RUARO, L.; Lima Neto, V. da C.; Motta, A.C.V. Efeito pH do solo e da concentração de inóculo no controle de Plasmodiophora brassicae. Summa Phytopathologica, v.36, n.1, p.16$20,2010$. 